\title{
Surgical treatment for advanced pancreatic cancer
}

\author{
Hyung Jun Kwon and Sang Geol Kim
}

\author{
Department of Surgery, Kyungpook National University Medical Center, Daegu, Korea
}

\begin{abstract}
The role of multimodality therapy and surgery for the treatment of locally advanced pancreatic cancer remains to be determined. Although no randomized trials have been done to determine the optimal management of this difficult clinical problem, numerous series reporting successful surgical resection with negative (R0) or microscopic margin (R1) showing favorable long-term survival provide a basis for an aggressive approach in selected cases of advanced cancer of the pancreas. In the absence of conclusive clinical trials, neoadjuvant treatment followed by surgical resection seems to be the optimal approach for locally advanced pancreatic cancers when the potential for surgical resection is suggested by preoperative high quality CT imaging. In particular, when the tumor is within the criteria for borderline resectable pancreatic cancer, efforts to achieve R0 resection are warranted. For those selected cases invading the hepatic artery and superior mesenteric artery, combined arterial resection and reconstruction may be performed to achieve R0 resection. Nonetheless, such a complex procedure should be balanced by a high rate of postoperative complications. In contrast, in cases of tumors invading the celiac axis, R0 resection by combined celiac axis resection can be performed without a high rate of postoperative complications. Survival benefit needs to be verified by further studies in the future. (Korean J Hepatobiliary Pancreat Surg 2012;16:89-92)
\end{abstract}

Key Words: Carcinoma; Pancreatic ductal; Resection; Borderline resectable

\section{INTRODUCTION}

To date, the prognosis of pancreas cancer is dismal. Unfortunately, less than $20 \%$ of pancreatic cancer patients are diagnosed at the resectable stage. The resection rate is only approximately $30 \%$, even at a high-volume center. In contrast, $25-30 \%$ of the patients are diagnosed with locally-advanced tumors in the absence of distant metastases at initial presentation. ${ }^{1}$ In general, tumors up to T3 stage (AJCC $7^{\text {th }}$ ed.) are considered to be resectable, but strict criteria to define resectability are still lacking. T4 tumors invading the celiac axis, hepatic artery, and superior mesenteric artery (SMA) are generally considered to be unresectable; however several authors have reported aggressive surgeries such as portal vein, arterial resection, and multivisceral resections in an attempt to increase the curability for the advanced pancreas cancer with favorable results in selected patients. ${ }^{2-6}$ The National Comprehensive Cancer Network (NCCN) guidelines and consensus statement recently published regarding borderline resectability and unresectability imply that limited involvement of vascular structure by tumor once regarded as a barrier to resection is no longer a contraindication to resection with curative intent. ${ }^{7}$ In this article, the recent consensus statement of resectability and the reports of surgical resection for highly selected locally advanced pancreas cancer are reviewed. In addition, we present our survival data according to the curability of resection and 4 cases invading the celiac axis, which were resected by a modified Appleby procedure.

\section{STAGING OF THE LOCALLY ADVANCED PANCREAS CANCER}

The seventh edition of AJCC-UICC TNM staging of pancreatic cancer has not changed from that of the sixth edition. Tumors involving the celiac axis and superior mesenteric artery are locally advanced tumors and are defined as T4. These T4 tumors are generally regarded as unresectable, as evident on preoperative CT scan. However,

Received: August 16, 2012; Revised: August 18, 2012; Accepted: August 20, 2012

Corresponding author: Sang Geol Kim

Department of Surgery, Kyungpook National University Medical Center, 474, Hakjeong-dong, Buk-gu, Daegu 702-210, Korea

Tel: +82-53-200-2708, Fax: +82-53-200-2027, E-mail: ksg@knu.ac.kr 
preoperative identification of major arterial invasion is not always accurate as reported by several radiologic studies. ${ }^{8-10}$ Thus, the risk of inadvertent exclusion from potentially curative resection should be considered in the management of patients with locally advanced pancreatic cancer. With the advances in high quality CT imaging, new concepts to define the extent of venous and arterial invasion have been developed and a subset of tumors called borderline resectable pancreas cancer (BRPC) that blur the distinction between resectable and locally advanced pancreatic cancer is categorized. ${ }^{11}$ BRPC, once considered to be unresectable, has now been accepted as a resectable tumor, although it has a high risk of developing into disseminated disease and for incomplete resection to occur. With neoadjuvant chemoradiotherapy for the BRPC patients, a considerable amount of patients have been reported to undergo complete resection. ${ }^{12}$

\section{NCCN CRITERIA TO DEFINE BORDERLINE RESECTABLE PANCREAS CANCER}

According to NCCN guideline, tumors considered BRPC include the following: (a) Having no distant metastases, (b) Venous involvement of the superior mesenteric vein (SMV)/portal vein showing tumor abutment with or without impingement and narrowing of the lumen, encasement of the SMV/portal vein without encasement of the nearby arteries, or short segment venous occlusion resulting from either tumor thrombus or encasement with suitable vessel proximal and distal to the area of vessel involvement allowing for safe resection and reconstruction.

(c) Gastroduodenal artery encasement up to the hepatic artery with either short segment encasement or direct abutment of the hepatic artery, without extension to the celiac axis. (d) Tumor abutment of the SMA not to exceed $180^{\circ}$ of the circumference of the vessel wall. In the cases of BRPC as described above, neoadjuvant chemotherapy have been reported to increase the resectability.

\section{NCCN CRITERIA TO DEFINE THE UNRESECTABLE TUMOR}

According to NCCN guidelines for pancreas cancer that have been published recently, the presence of distant metastasis, unreconstructible SMV/Portal invasion, SMA, or celiac axis encasement of more than $180^{\circ}$ are criteria for unresectable pancreas head, body, and tail cancer. In particular, any celiac abutment is a criterion for unresectability for pancreatic head cancer. ${ }^{11}$

\section{CT FINDINGS SUGGESTING A TUMOR IS AMENABLE TO VASCULAR RECONSTRUCTION}

A tumor in the head of the pancreas with non-circumferential apposition to the portal vein is usually resectable; non-circumferential invasion of the SMV, proximal to jejunal branches is usually indicative of resectability. Follow-up CT may be used to assess response to neoadjuvant chemoradiotherapy when baseline CT shows the tumor abutting $\left(<180^{\circ}\right.$ of circumferential contact) a short segment, typically less than $2 \mathrm{~cm}$, of the superior mesenteric or hepatic artery. CT scans are usually obtained at 4 to 6 -week intervals. The principal evidence of potential resectability is the disappearance of soft tissue abutting the arteries. Persistent periarterial stranding does not preclude surgery, because this finding may be the result of radiation therapy. ${ }^{13}$

\section{CT FINDINGS SUGGESTING A TUMOR IS NOT AMENABLE TO VASCULAR RECONSTRUCTION}

Circumferential involvement of a superior mesenteric vein-portal vein segment more than $2 \mathrm{~cm}$ long, thrombus in the vein, or invasion of the transverse mesocolon indicate non-resectability; usually, vein reconstruction is not attempted in such cases. Circumferential involvement of a short segment, typically less than $2 \mathrm{~cm}$, of the SMV close to the portal confluence is considered borderline resectable. The main concern for operating on these patients is that the tumor will be closely applied to the retroperitoneal margin. Invasion of the transverse mesocolon precludes curative surgery, because adequate control of the venous tributaries making up the SMV is virtually impossible. Invasion of the gastroduodenal artery per se is not a contraindication to surgery because this vessel is resected during pancreaticoduodenectomy. Celiac, hepatic, or superior mesenteric arterial occlusion and circum- 
ferential invasion remain contraindications to curative surgery. ${ }^{13}$

\section{EXPERT PANEL CONSENSUS STATEMENT REGARDING THE MARGIN STATUS AFTER PANCREATICODUODENECTOMY AND ROLE OF "PALLIATIVE PANCREATICODUODENECTOMY"}

Evans et al. published an expert panel consensus statement regarding important issues that may influence the survival of the pancreatic cancer patients. ${ }^{7}$ Regarding the margin status, they described that in the most contemporary series (in the setting of regular multimodality therapy, and no $\mathrm{R} 2$ resections), R1 resection with median survival as high as 22 months was not a predictive factor for survival on multivariate analysis.

The expert group stated that safe achievement of an R0 margin is the main surgical objective of pancreaticoduodenectomy, as it is of great importance for extended survival. The SMA margin is the most important driver of this outcome. The impact of $\mathrm{R} 1$ resection on ultimate clinical outcome is uncertain, but multimodality therapy may recover an R1 margin and improve survival to that similar to R0 resections. ${ }^{7}$ Regarding "the Palliative pancreaticoduodenectomy", the expert group stated that R0 resection was not accomplished in a substantial proportion of patients, even at major medical centers, and those cases end up with R1 and even R2 resections. Some studies demonstrate that a margin positive resection may yield a survival and quality-of-life advantage compared with standard surgical bypass. However, there is no role for "palliative resection" of pancreatic cancer in the setting of metastatic or preoperatively apparent, locally extensive disease. $^{7}$

\section{THE ROLE OF ARTERIAL RESECTION FOR THE TUMORS INVADING COMMON HEPATIC ARTERY AND SUPERIOR MESENTERIC ARTERY}

As described in NCCN guidelines, any tumors invading the common hepatic artery and superior mesenteric artery are beyond the criteria for resectability. Thus, arterial re- section, particularly in cases of tumor extension to the superior mesenteric artery (SMA) or common hepatic artery, has traditionally been considered a contraindication to pancreaticoduodenectomy. The reason for unresectability in such cases may be a lack of survival benefit in spite of a high risk of mortality and morbidity associated with a complex surgical procedure. However, modern surgical refinement decreased complications after pancreatic surgery and an aggressive surgical approach such as portal vein, arterial resection, and multivisceral resections in anecdotal cases have yielded favorable survival comparable to that of resectable tumors. Bockhorn et al. reported that the median survival of 29 patients who underwent arterial en bloc resection was similar to the patient who did not need arterial resection (14.0 versus 15.8 months; $p=0.152$ ) and was better than the patient who underwent palliative bypass (7.5 months; $p=0.028) .{ }^{14}$ In another series, Philippe et al. reported similar results; 1 - and 3-year survival rates were similar in the patients who underwent arterial resection (65.9\% and $22.1 \%$, median 17 months) compared to the matched control $(50.0 \%$ and $17.6 \%$, median 12 months; $p=0.581){ }^{15}$ Nevertheless, higher morbidity and mortality rates associated with arterial resection remain to be overcome.

\section{ROLE OF ARTERIAL RESECTION FOR THE TUMORS INVADING CELIAC AXIS}

Tumors of the head of the pancreas usually involve the nerve plexus around the SMA and common hepatic artery and combined resection of the involved artery require reconstruction. In contrast, tumors of the body of the pancreas spread towards the celiac plexus, celiac axis, and common hepatic artery. Combined en bloc resection of these structures does not require either arterial or pancreatobiliary-gastrointestinal reconstruction. Thus, this procedure does not have much higher rate of post-operative mortality and morbidity. Hirano et al. reported that the 23 patients who had undergone celiac axis resection showed a higher rate of $\mathrm{R} 0$ resection and a favorable 1 -year and 5-year survival rates $(71 \%$ and $42 \%$, respectively). ${ }^{16}$ The authors concluded that distal pancreatectomy with celiac axis resection for locally advanced pancreatic body cancer is feasible, safe, and has the potential to achieve complete local control. 


\section{SURVIVAL OF PANCREAS CANCER PATIENT AT KYUNGPOOK NATIONAL UNIVERSITY HOSPITAL AND FOUR CASES OF PANCREAS CANCER INVADING CELIAC AXIS TREATED BY DISTAL PANCREATECTOMY AND CELIAC AXIS RESECTION}

From February 2000 to December 2010, 123 patients with pancreas cancer underwent surgical surgery at Kyungpook National University Hospital (KNUH). R0, $\mathrm{R} 1, \mathrm{R} 2$, and no resection were done in $46.3 \%, 5.7 \%, 4.8 \%$ and $43.1 \%$ (57, 7, 3, and 53 patients out of 123 patients), respectively. The R0 resection rate was $46.3 \%$. The median survival of R0, R1, and R2 was 43.9 months, 22.6 months, and 8.0 months, respectively $p<0.05$ ). The survival of R2 resection group was not different from the non-resection group. Recently, we performed distal pancreatectomy with celiac axis resection for the 4 patients with pancreatic cancer that was invading the celiac axis. On CT imaging, invasion to the celiac artery and splenic artery was evident in all cases. Distal pancreatectomy with celiac resection was performed with subtotal gastrectomy in one case and with preservation of the stomach in the remaining 3 cases. R0 resection was possible in 3 cases, except for in 1 case which had a positive para-aortic lymph node invading left renal vein. Three patients with R0 resection are free from tumor recurrence for 14,4 , and 3 months, respectively. These patients should be followed up longer to determine the effect on survival. The patient with $\mathrm{R} 1$ resection had liver metastasis at 12 months after surgery and is alive 15 months after surgery.

\section{REFERENCES}

1. Cancer Institute: Surveillance, Epidemiology, and End Results
(SEER). http://seer.cancer.gov/faststats/.

2. Fortner JG, Kim DK, Cubilla A, et al. Regional pancreatectomy: en bloc pancreatic, portal vein and lymph node resection. Ann Surg 1977;186:42-50.

3. Bold RJ, Charnsangavej C, Cleary KR, et al. Major vascular resection as part of pancreaticoduodenectomy for cancer: radiologic, intraoperative, and pathologic analysis. J Gastrointest Surg 1999;3:233-243.

4. Sasson AR, Hoffman JP, Ross EA, et al. En bloc resection for locally advanced cancer of the pancreas: is it worthwhile? J Gastrointest Surg 2002;6:147-157.

5. Tseng JF, Raut CP, Lee JE, et al. Pancreaticoduodenectomy with vascular resection: margin status and survival duration. $\mathrm{J}$ Gastrointest Surg 20048:935-949.

6. Nakano H, Bachellier P, Weber JC, et al. Arterial and vena caval resections combined with pancreaticoduodenectomy in highly selected patients with periampullary malignancies. Hepatogastroenterology 2002;49:258-262.

7. Evans DB, Farnell MB, Lillemoe KD, et al. Surgical treatment of resectable and borderline resectable pancreas cancer: expert consensus statement. Ann Surg Oncol 2009;16:1736-1744.

8. Karmazanovsky G, Fedorov V, Kubyshkin V, et al. Pancreatic head cancer: accuracy of $\mathrm{CT}$ in determination of resectability. Abdom Imaging 2005;30:488-500.

9. Li H, Zeng MS, Zhou KR, et al. Pancreatic adenocarcinoma: the different CT criteria for peripancreatic major arterial and venous invasion. J Comput Assist Tomogr 2005;29:170-175.

10. Brügel M, Rummeny EJ, Dobritz M. Vascular invasion in pancreatic cancer: value of multislice helical CT. Abdom Imaging 2004;29:239-245.

11. Tempero MA, Arnoletti JP, Behrman S, et al; NCCN Pancreatic Adenocarcinoma. Pancreatic adenocarcinoma. J Natl Compr Canc Netw 2010;8:972-1017.

12. Pingpank JF, Hoffman JP, Ross EA, et al. Effect of preoperative chemoradiotherapy on surgical margin status of resected adenocarcinoma of the head of the pancreas. J Gastrointest Surg 2001;5:121-130

13. Lall CG, Howard TJ, Skandarajah A, et al. New concepts in staging and treatment of locally advanced pancreatic head cancer. AJR Am J Roentgenol 2007;189:1044-1050.

14. Bockhorn M, Burdelski C, Bogoevski D, et al. Arterial en bloc resection for pancreatic carcinoma. Br J Surg 2011;98:86-92.

15. Bachellier P, Rosso E, Lucescu I, et al. Is the need for an arterial resection a contraindication to pancreatic resection for locally advanced pancreatic adenocarcinoma? A case-matched controlled study. J Surg Oncol 2011;103:75-84.

16. Hirano S, Kondo S, Hara $\mathrm{T}$, et al. Distal pancreatectomy with en bloc celiac axis resection for locally advanced pancreatic body cancer: long-term results. Ann Surg 2007;246:46-51. 